\title{
Offline calibration procedure of the CMS Drift Tube detectors
}

G. Abbiendi, ${ }^{a}$ N. Amapane, ${ }^{b}$ C. Battilana, ${ }^{a}$ R. Bellan, ${ }^{c}$ P. Biallass,${ }^{d}$ M. Biasotto, ${ }^{e}$ S. Bolognesi, ${ }^{b, 1}$ A. Calderon Tazon, ${ }^{f}$ F.R. Cavallo, ${ }^{a}$ M. Cepeda,${ }^{g}$ G. Cerminara, ${ }^{b, c}$

B. De La Cruz ${ }^{g}$ C. Diez Pardos, ${ }^{g}$ C. Fernandez Bedoya,${ }^{g}$ J. Fernandez Menendez, ${ }^{h}$ M.C. Fouz Iglesias,${ }^{g}$ J. Frangenheim, ${ }^{d}$ M. Giunta, ${ }^{a}$ A. Gresele,${ }^{i}$ L. Guiducci, ${ }^{a}$ M. Gulmini, ${ }^{e}$ K. Hoepfner ${ }^{d}{ }^{d}$ M.I. Josa Mutuberria,${ }^{g}$ S. Lacaprara, ${ }^{e}$ S. Marcellini, ${ }^{a}$ P. Martinez Ruiz Del Arbol, ${ }^{j}$ S. Maselli, ${ }^{b}$ G. Masetti, ${ }^{a, c}$ A.T. Meneguzzo, ${ }^{f}$ G. Mila, ${ }^{b}$ J.A. Molina Insfran, ${ }^{g}$ M. Passaseo,${ }^{f}$ A. Perrotta, ${ }^{a}$ J. Puerta Pelayo, ${ }^{g}$ H. Reithler,${ }^{d}$ P. Ronchese, ${ }^{f}$ T. Rovelli, ${ }^{a}$ J. Santaolalla Camino, ${ }^{g}$ D. Teyssier, ${ }^{d}$ R. Travaglini, ${ }^{a}$ D. Trocino, ${ }^{b}$ S. Vanini, ${ }^{f}$ S. Ventura, ${ }^{f}$ A. Vilela Pereira ${ }^{b}$ and M. Zanetti ${ }^{c}$

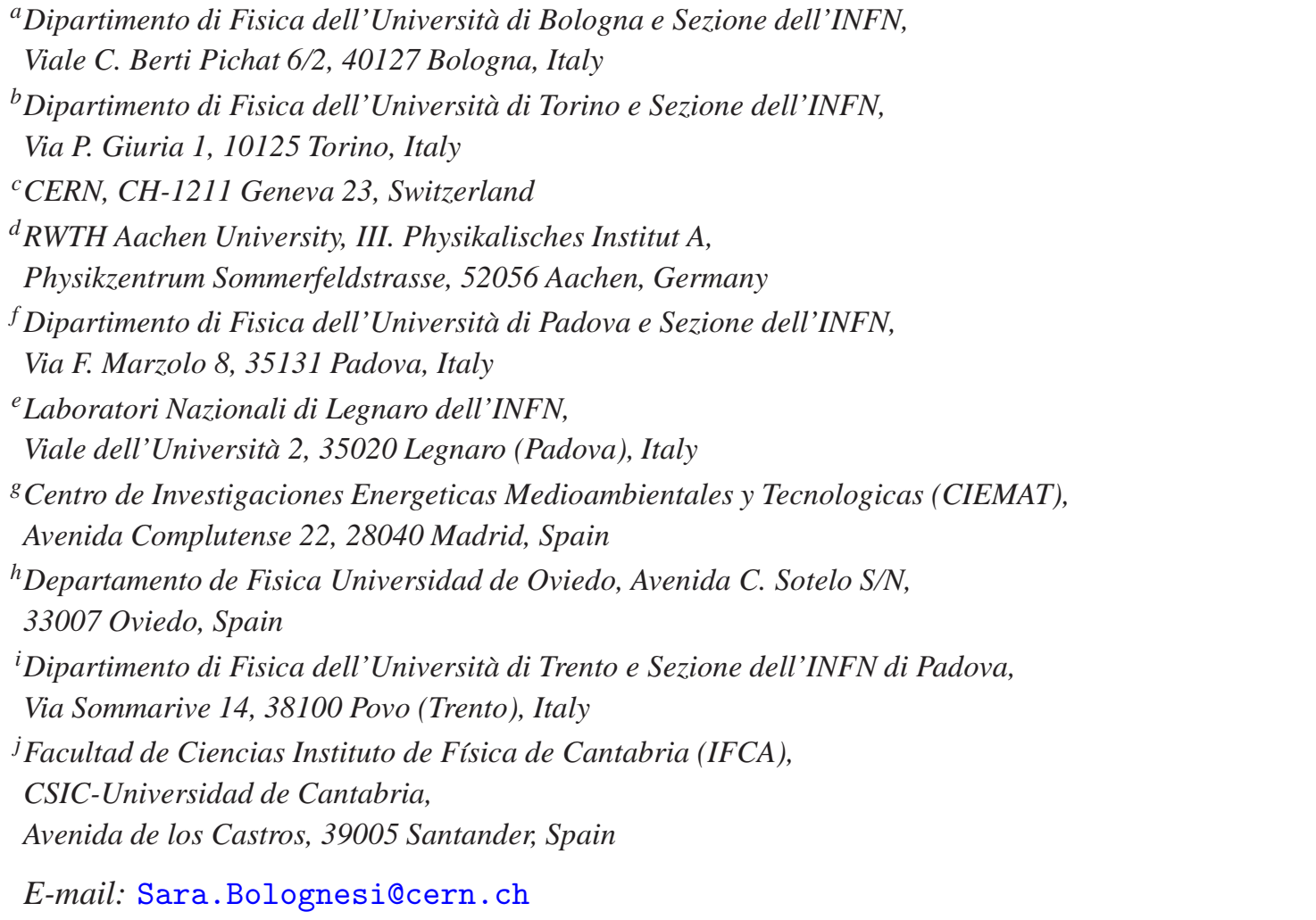

\footnotetext{
${ }^{1}$ Corresponding author.
} 
AbStRact: The barrel region of the Compact Muon Solenoid (CMS) experiment at the Large Hadron Collider is instrumented with Drift Tube (DT) detectors. This paper describes in full details the calibration of the DT hit reconstruction algorithm.

After inter-channel synchronization has been verified through the appropriate hardware procedure, the time pedestals are extracted directly from the distribution of the recorded times. Further corrections for time-of-flight and time of signal propagation are applied as soon as the threedimensional hit position within the DT chamber is known. The different effects of the time pedestal miscalibration on the two main hit reconstruction algorithms are shown.

The drift velocity calibration algorithm is based on the meantimer technique. Different meantimer relations for different track angles and patterns of hit cells are used. This algorithm can also be used to determine the uncertainty on the reconstructed hit position.

KEYWORDS: Wire chambers(MWPC, Thin-gap chambers, drift chambers, drift tubes, proportional chambers etc); Pattern recognition, cluster finding, calibration and fitting methods 


\section{Contents}

1 Introduction 1

2 Calibration of the time pedestals 3

2.1 Determination of the $t_{\text {trig }}$ offset 4

3 Calibration of the drift velocity 6

3.1 Meantimer technique 9

$\begin{array}{ll}3.2 & \text { Estimate of the cell resolution } \\ & 10\end{array}$

4 Interplay of meantimer computation and time pedestals determination 11

5 Conclusions 12

\section{Introduction}

The barrel region of the CMS detector is equipped with a system of Drift Tube (DT) chambers [1] arranged in the return yoke of the solenoidal magnet [2].

The basic element of a DT detector is the drift cell, whose section is shown in figure 1 . The cell has a transverse size of $42 \times 13 \mathrm{~mm}^{2}$ with a $50 \mu \mathrm{m}$ diameter stainless steel anode wire at the centre. The gas is a $85 \% / 15 \%$ mixture of $\mathrm{Ar} / \mathrm{CO}_{2}$, which provides good quenching properties and a saturated drift velocity of about $54 \mu \mathrm{m} / \mathrm{ns}$. The maximum drift time is therefore $\approx 390 \mathrm{~ns}$, i.e. 15 LHC bunch crossings.

Four staggered layers of parallel cells form a superlayer. A chamber consists of two superlayers measuring the $R \phi$ coordinates, with the wires parallel to the beam line, and an orthogonal superlayer measuring the $R Z$ coordinates. A schematic view of a chamber is shown in figure 2 .

Charged particles crossing a cell produce ionization electrons in the $\mathrm{Ar} / \mathrm{CO}_{2}$ gas mixture; the drift time of such electrons in the electrostatic field is measured to determine the spatial coordinate
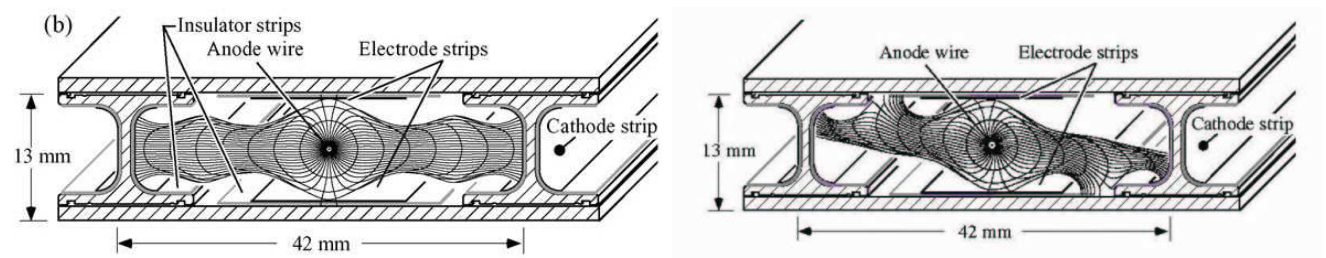

Figure 1. Transverse view of a Drift Tube cell in the barrel. The drift lines and the isochrones for a typical voltage configuration of the electrodes and representative gas mixture are shown for the case of zero magnetic field (left) and a $0.45 \mathrm{~T}$ magnetic field parallel to the anode wires (right). 


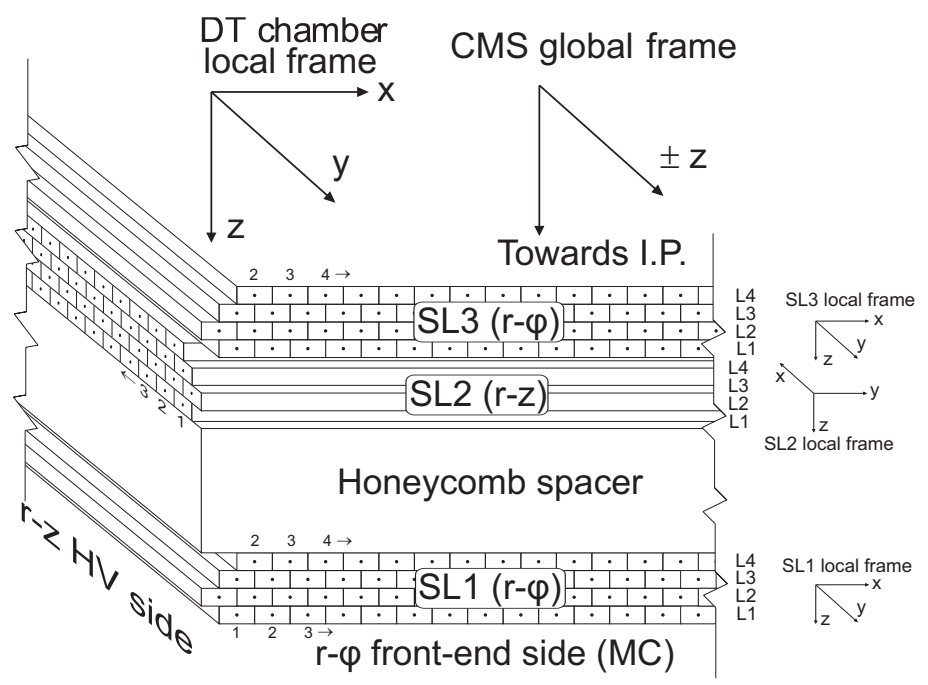

Figure 2. Schematic view of a DT chamber, showing the conventions on superlayer, layer and wire numbering and the orientation of reference frames.

of the ionizing particle. Electrons produced at a time $t_{\text {ped }}$ by the incoming particle migrate toward the anode with a velocity $v_{\text {drift }}$ and reach the anode at a time $t_{T D C}$, which is the time measured by the TDC. The distance of the track with respect to the anode wire is therefore given by

$$
x=\int_{t_{\mathrm{ped}}}^{t_{T D C}} v_{\mathrm{drift}} \cdot d t
$$

The measurement of the track distance from the wire $(x)$ requires understanding of this timespace relationship.

Two reconstruction algorithms are available in the CMS software to convert the measured time into a hit position. The first reconstruction algorithm is based on the assumption of a constant drift velocity within the entire cell. In this case, the above formula becomes

$$
x=\left(t_{T D C}-t_{\mathrm{ped}}\right) \cdot v_{\mathrm{drift}}^{E F F}=t_{\mathrm{drift}} \cdot v_{\mathrm{drift}}^{E F F}
$$

where $v_{\text {drift }}^{E F F}$ is the effective, average drift velocity.

The goal of the calibration procedure is in this case to determine the time pedestal $\left(t_{\text {ped }}\right)$, which is needed to extract the drift time $\left(t_{\text {drift }}\right)$ from the TDC measurement $\left(t_{T D C}\right)$, and the average drift velocity $v_{\text {drift }}^{E F F}$.

The value of $v_{\text {drift }}^{E F F}$ depends on the track impact angle and on the residual magnetic field. However, the detector can be subdivided in properly limited spatial regions where these parameters can be assumed approximately constant. The calibration procedure is performed with the correspondent granularity, therefore the computed drift velocity is averaged under local variations of such parameters in each region. 
The second reconstruction algorithm is based on a parameterization of the cell response [3] obtained with GARFIELD [4]. This parameterization includes the dependence on the track impact angle, $\alpha$, and on the stray magnetic field $\mathbf{B}$ :

$$
x=f\left(\left(t_{T D C}-t_{\mathrm{ped}}\right), \alpha, \mathbf{B}\right)
$$

In this case the only quantity to be calibrated is $t_{\text {ped }}$, as the dependency on the relevant parameters is already accounted for by the parameterization.

It should be noted that the residual magnetic field and the track angle also influence the intrinsic cell resolution due to their effect on the cell non-linearities. Correct estimation of the hit uncertainty is important for the track fit; for this reason, the calibration algorithm must also be able to assign a correct uncertainty to the reconstructed hits.

The procedure to determine the time pedestals is described in section 2. Section 3 introduces the calibration of the drift velocity and the assignment of the uncertainty on the hit position. Finally, section 4 outlines the reciprocal dependence between the time pedestal and the drift velocity.

\section{Calibration of the time pedestals}

A DT measurement consists of a TDC time, which also contains other contributions besides the drift time of the ionization electrons in the cell, including

- the time-of-flight (TOF) of the muon from the interaction point to the cell;

- the propagation time of the signal along the anode wire;

- delays due to the cable length and read-out electronics;

- the time latency due to the trigger electronic chain.

These offsets must be estimated and subtracted from the TDC time during reconstruction. The jitter in the drift time deriving from the uncertainties of this procedure directly contributes to the DT resolution.

The extraction of the drift time from the TDC measurement is performed in several consecutive steps.

- Inter-channel synchronization.

First, it is necessary to correct the measured TDC times for the relative difference in the signal path length to the readout electronics of each wire. This relative difference is measured for each wire by sending simultaneous (within an error smaller than $150 \mathrm{ps}$ ) "test-pulses" to the front-end electronic. Then the difference between the measured times, called $t_{0}$, is computed. This relative correction is usually between 1 and $8 \mathrm{~ns}$. Once the $t_{0}$ is subtracted, the resulting TDC times for the different channels within the chamber are synchronized relative to each other.

- Absolute offset determination.

Once the channels are synchronized, it is possible to compute the absolute offset of the drift 
time distribution. This offset, called $t_{\text {trig }}$ because of its dependence on the trigger latency, allows the extraction of the drift time from the TDC measurement. The $t_{\text {trig }}$ is directly estimated from the distribution of the recorded times using the procedure described in section 2.1. Its value depends on the specific DAQ setup and is usually on the order of a few $\mu \mathrm{s}$.

Note that the determination of these two delays does not completely solve the problem of synchronization of the measured times. In fact, due to the limited available data, the $t_{\text {trig }}$ is usually computed for a group of cells together, e.g., all cells in a superlayer. In this case, the measured $t_{\text {trig }}$ includes the average TOF and the average signal propagation time of the muons that crossed the superlayer. If the chamber is uniformly illuminated, which is the case for pp-collisions, this average TOF is approximately equivalent to that of a muon crossing the superlayer center, while the average signal propagation time is equivalent to the propagation time for a signal produced in the middle of the wire.

Therefore, further corrections for these two effects can be computed as soon as the threedimensional hit position within the chamber is known, namely after the hits are associated into 3D track segments. Specifically, if the $t_{\text {trig }}$ is computed for a full, uniformly illuminated superlayer:

- the 3D position obtained from the segment extrapolation to the hit plane, if available, is used to correct the TOF with respect to the superlayer center;

- the hit coordinate along the wire is used to correct the propagation time with respect to the middle of the wire, assuming a propagation velocity of $0.244 \mathrm{~m} / \mathrm{ns}$, as directly measured on test-beam data [5].

These corrections can be as high as $\approx 4$ ns for the TOF and $\approx 6$ ns for the signal propagation delay, they can be adopted or switched off in case of different running conditions. For instance, this is the case of cosmic data, where the previous definition of the TOF can not be applied, or of test-beam data, where the chamber is usually illuminated in a relatively small region. Particular care has been taken to provide enough flexibility for such cases.

\subsection{Determination of the $t_{\text {trig }}$ offset}

Since the measured TDC times of the different channels in a chamber have already been synchronized by subtracting the $t_{0}$ offset, the $t_{\text {trig }}$ can be computed with every possible granularity within the chamber. The usual choice is to compute it superlayer by superlayer, as a compromise between the accuracy in accounting for the average TOF and the quantity of available data.

Due to its dependency on the trigger latency, the $t_{\text {trig }}$ pedestal must be calibrated each time the trigger configuration and synchronization change. The $t_{\text {trig }}$ also depends on the running conditions, as it accounts for the average contribution of the TOF and the signal propagation along the anode wire.

The pedestal can be estimated directly from the distribution of the recorded times, which is usually referred as the time box. An example of such distribution is shown in figure 3 for a superlayer $R Z$ of a chamber exposed to a muon test beam.

In order to compute the pedestal it is necessary to find a feature of this distribution which can be identified in an unambiguous and automatic way. Earlier studies have shown that a suitable 

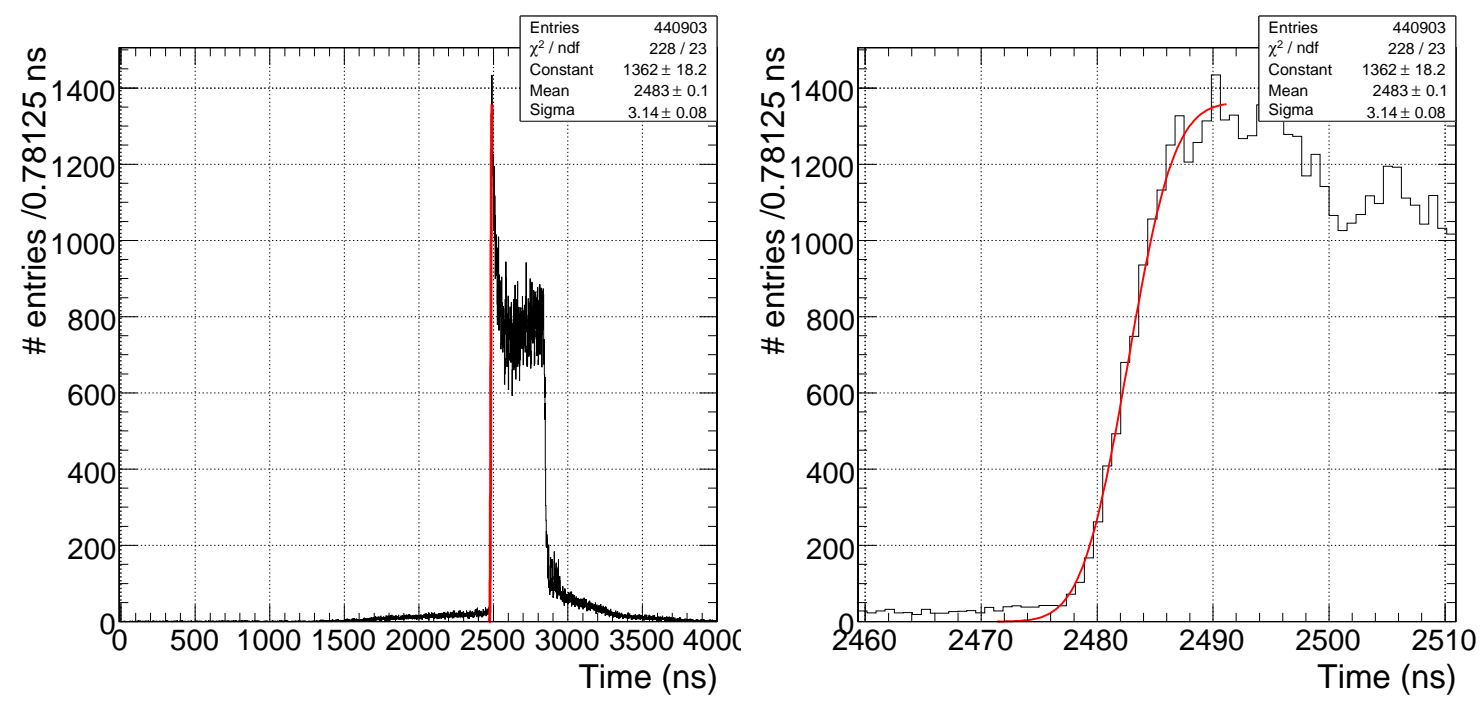

Figure 3. Distribution of the recorded times of one superlayer acquired during the 2004 test beam. The rising edge of the time box is fitted with the integral of a Gaussian to measure the time pedestal of the drift times $\left(t_{\text {trig }}\right)$.

feature is the inflexion point of the rising edge, which can be obtained from a Gaussian fit of the derivative of the time-box distribution [6]. This method, however, is sensitive to noise and spikes due to the read-out electronics. To implement an automatic procedure to fit the drift time box in an unattended mode for all the superlayers of the 250 DT chambers, we developed a different, more robust method, based on a fit of the rising edge of the drift time distribution with the integral of the Gaussian function (the so-called error function):

$$
f(t)=\frac{1}{2} I\left[1+\operatorname{erf}\left(\frac{t-\langle t\rangle}{\sigma \sqrt{2}}\right)\right]
$$

where the normalization $I$, the standard deviation $\sigma$ and the mean $\langle t\rangle$ are free parameters of the fit. In figure 3 an example of this fit is shown for a time box of a $R Z$ superlayer illuminated during a muon test beam.

The inflexion point of the rising edge of the time box, $\langle t\rangle$, does not directly represent the time pedestal of the distribution, but can be related to it by defining

$$
t_{\text {trig }}=\langle t\rangle-k \cdot \sigma
$$

where $k$ is a factor that is tuned requiring the minimization of the residuals on the reconstructed hit position, superlayer by superlayer. A typical value of the $k$ factor is 1.3.

In order to obtain meaningful residual distributions, it is necessary to have a preliminary estimation of the $t_{\text {trig }}$ with a resolution of at least $10 \mathrm{~ns}$, while the value of the $t_{\text {trig }}$ can vary up to some microseconds, depending on the trigger configuration and cable length. Therefore the fit of the time box rising edge has to be performed before the $k$ factor optimization can be done.

It should be noted that the optimal value of $t_{\text {trig }}$ depends on the algorithm used in the reconstruction. In particular, the cell parameterization has a small arbitrary intrinsic offset deriving from 
the way the signal arrival time is computed in the GARFIELD simulation [3]. For this reason a fine tuning of the $t_{\text {trig }}$ has to be done differently for the two reconstruction algorithms.

In addition, the effect of a mis-calibration of the time pedestal is different for the two reconstruction algorithms. If the reconstruction is performed using a constant drift velocity over the entire cell, a not perfectly calibrated $t_{\text {trig }}$ results in an error on the estimated drift time and therefore in a constant offset for all the reconstructed distances from the wire. This is illustrated for Monte Carlo simulated pp-collisions in figure 4 which shows the residuals on the distance from the wire $\left(\left|x_{\text {reco }}\right|-\right.$ $\left.\left|x_{\text {sim }}\right|\right)$ for two particular choices of the time pedestal: the "optimal" value and a $t_{\text {trig }} 6 \mathrm{~ns}$ larger than the optimal one. ${ }^{1}$ The error on the pedestal affects the mean value of the distribution of a quantity given by $-\Delta t \cdot v_{\text {drift }}$, while the standard deviation of the Gaussian fit is essentially unaffected, being dominated by the non-linearities responsible for the modulation shown in the scatter plots of figure 4. This independence of $\sigma$ on the actual value of $t_{\text {trig }}$ allows $t_{\text {trig }}$ to be optimized superlayer by superlayer by tuning the $k$ factor of eq. (2.2) to minimize the mean of the residual distribution.

Note that figure 4 shows the distributions obtained for all the muon tracks recorded in the $R Z$ superlayers in the high pseudorapidity region $(0.6<|\eta|<1.2)$, where the effects of nonlinearity are expected to be larger. In fact these effects increase with the track incident angles relative to the direction normal to the chambers and with the residual magnetic field in the chamber volume.

The effect of a mis-calibration of the $t_{\text {trig }}$ pedestal is more complex when the reconstruction is performed using the GARFIELD parameterization. As this parameterization accounts for the cell non-linearity as a function of the drift time, an offset in the input time does not simply produce an offset in the mean value of the residuals, but also implies that the non-linearities are accounted for incorrectly, resulting in a wider residual distribution. This is illustrated in figure 5, which again shows the residuals of the reconstructed hit distances from the wire in the $R Z$ superlayers in the high pseudorapidity region $(0.6<|\eta|<1.2)$ for the two extreme choices of the $t_{\text {trig }}$ pedestal considered above. It can be observed that since the parameterization corrects for the non-linearities, the presence of an offset in the $t_{\text {trig }}$ introduces artificial deviations, leading to a broadening of the residual distribution in addition to a shift of the mean value. This effect can be used for the optimization of the $t_{\text {trig }}$ value, which can be performed by minimizing the residuals: the optimal $t_{\text {trig }}$ value is the value for which the parameterization of non-linearities best fits the input data.

It should be noted that in real data the residuals will be computed with respect to the reconstructed 3D segment and this will introduce systematic effects on the $k$ factor optimization to be studied.

\section{Calibration of the drift velocity}

The drift velocity depends on many parameters, including the gas purity and conditions and the electrostatic configuration of the cell. Moreover, the presence of stray magnetic field and the angle of incidence of the track (indicated with $\alpha$ in figure 6) influence the effective drift velocity.

The working condition of the chambers will be monitored continuously and important variations are not expected among different regions of the spectrometer. The situation is different for the

\footnotetext{
${ }^{1}$ This value of the pedestal corresponds to an extreme case of mis-calibration, chosen for illustration purposes. The $t_{\text {trig }}$ can be usually calibrated with much higher accuracy.
} 

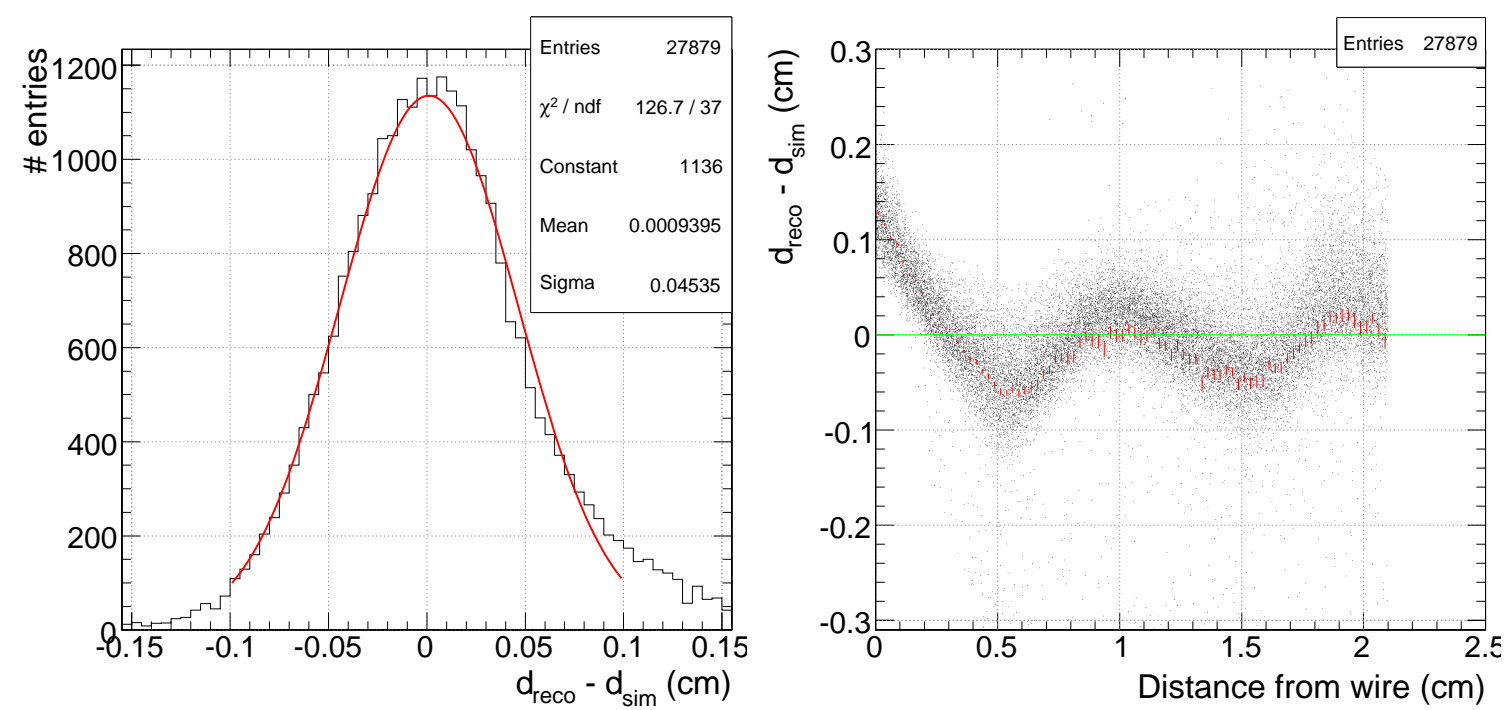

(a) Optimal time pedestal $t_{\text {trig }}$ (MC truth)
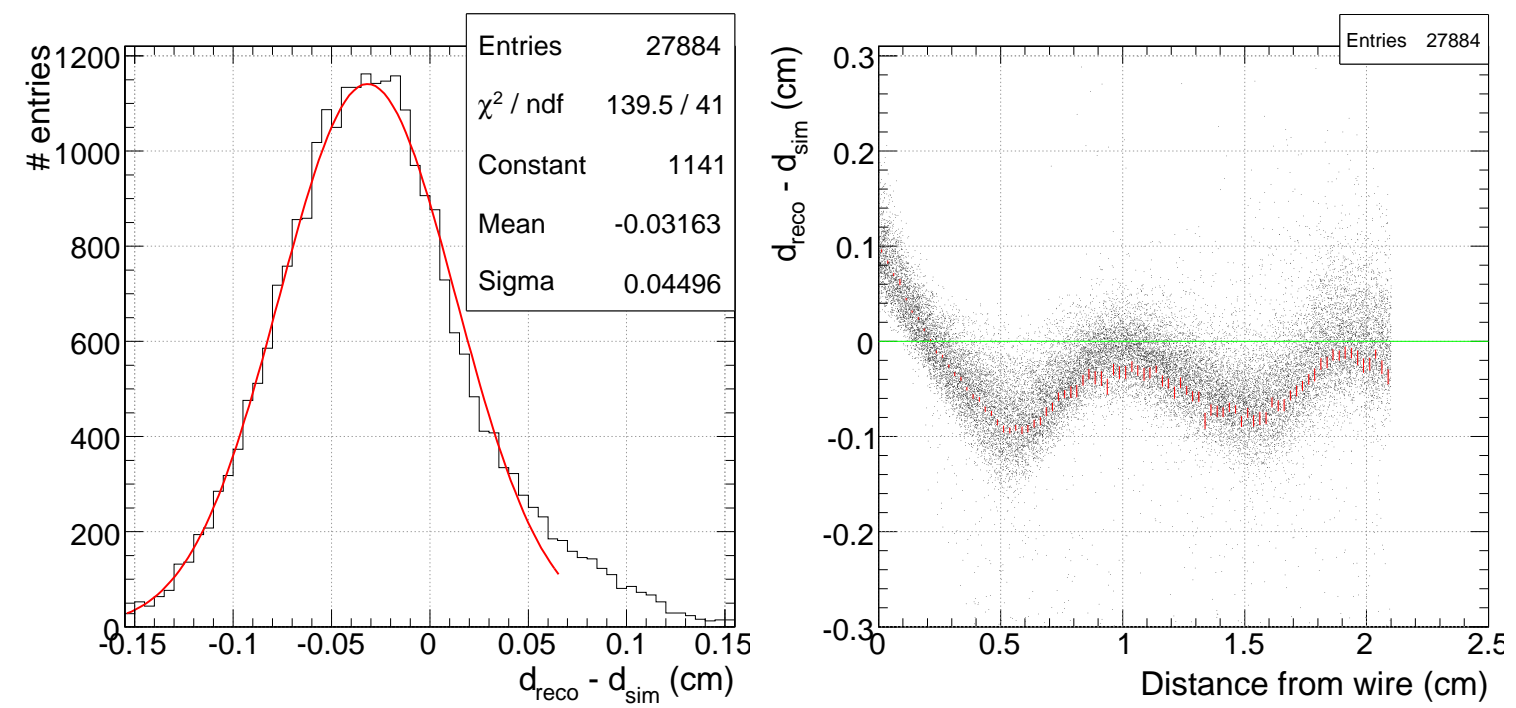

(b) $t_{\text {trig }} 6$ ns larger than the optimal one

Figure 4. Residuals between the reconstructed and the simulated hit distances from the wire $(d=|x|)$ for $R Z$ superlayers in the high pseudorapidity region $(0.6<|\eta|<1.2)$. The plots on the right show the residuals as a function of the distance from the wire. The plots have been obtained using a constant drift velocity with the optimal value of the $t_{\text {trig }}$ (a) and with a $t_{\text {trig }} 6 \mathrm{~ns}$ larger than the optimal one (b). No further correction for the TOF or the time of signal propagation along the wire has been applied.

stray magnetic field and for the track impact angle: these parameters will vary substantially moving from chamber to chamber and also from superlayer to superlayer due to the different positions within the return yoke and the different pseudorapidities of the impact angles in the $R Z$ cells. In particular the effect of the track angle is due to the fact that the electrons with smaller drift time are not the ones produced in the cell median plane. This effect has been studied [1]; in particular an increase of the drift velocity of about $7 \%$ has been observed for an impact angle of 50 degrees. 

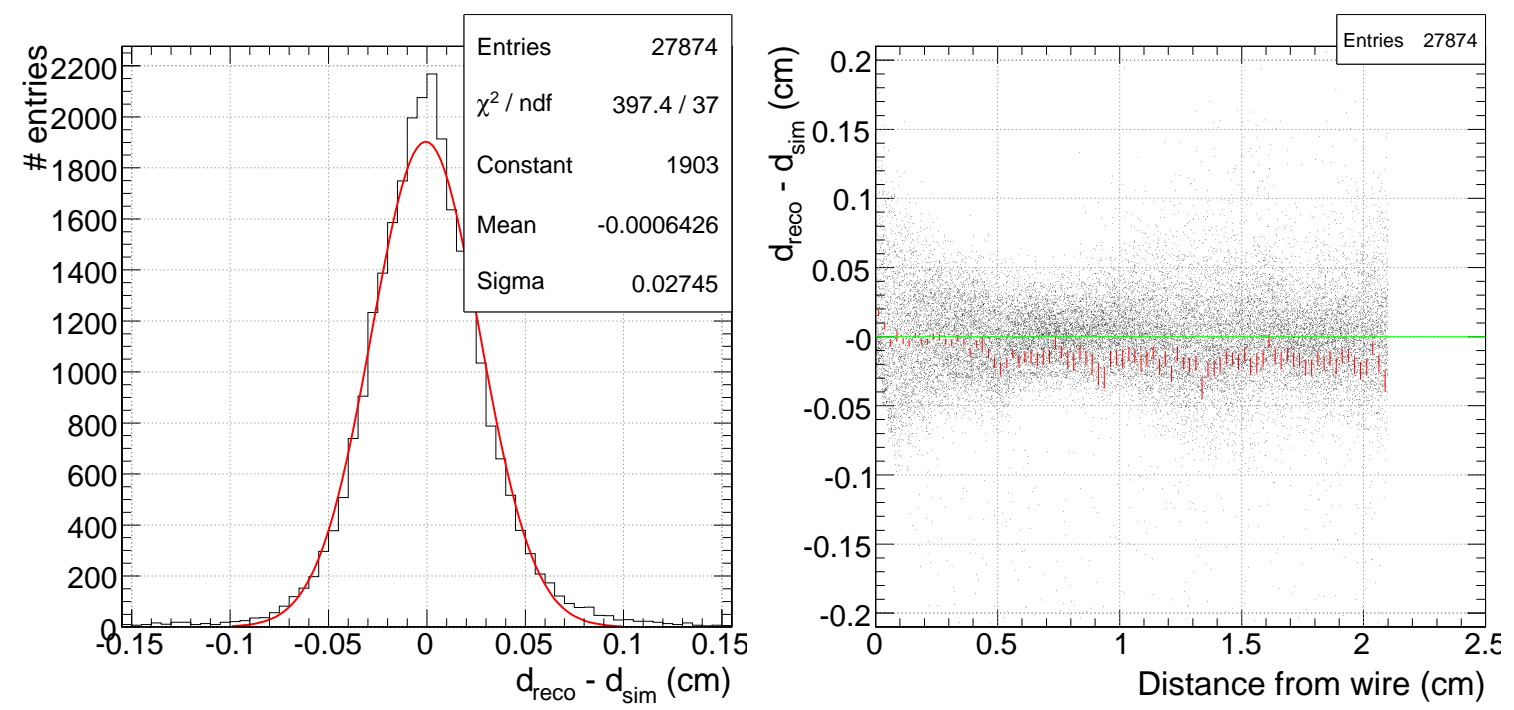

(a) Optimal time pedestal $t_{\text {trig }}$ (MC truth)
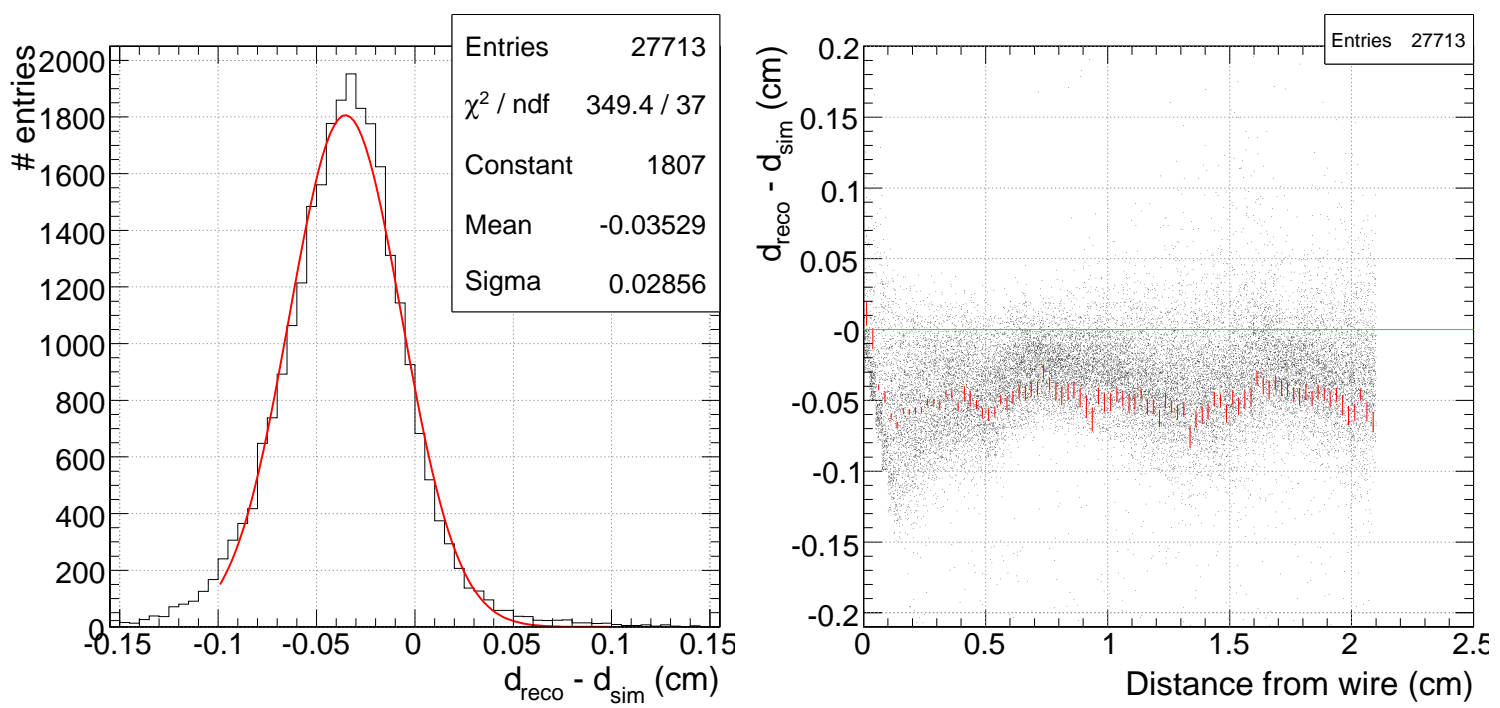

(b) $t_{\text {trig }} 6$ ns larger than the optimal one

Figure 5. Residuals between the reconstructed and the simulated hit distances from the wire $(d=|x|)$ for $R Z$ superlayers in the high pseudorapidity region $(0.6<|\eta|<1.2)$. The plots on the right show the residuals as a function of the distance from the wire. The plots have been obtained using the GARFIELD parameterization with the optimal value of the $t_{\text {trig }}$ (a) and with a $t_{\text {trig }} 6 \mathrm{~ns}$ larger than the optimal one (b). No further correction for the TOF or the time of signal propagation along the wire has been applied.

For this reason, the average drift velocity must be calibrated for different groups of cells separately, chosen so that within each group the magnetic field and the track angle are approximately uniform.

To fulfill these requirements, a calibration algorithm based on the so-called meantimer [7] computation has been developed and is described below. This technique estimates the maximum drift time and therefore the average drift velocity in the cell. Moreover, it also measures the cell resolution, which can be used as an estimate of the uncertainties associated to each measurement. 


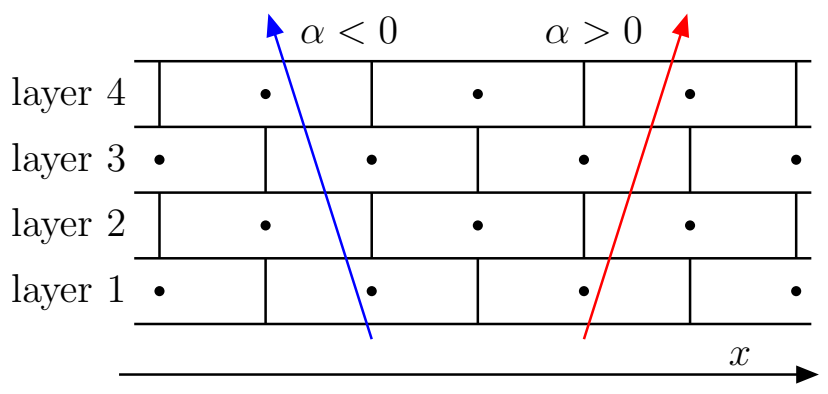

Figure 6. Schematic of a superlayer showing the track segment angle convention and the pattern of semicells crossed by the track.

\subsection{Meantimer technique}

The meantimer formulas are relations among the drift times produced by a track in consecutive layers of a superlayer $\left(t_{i}\right)$ and the maximum drift time $\left(T_{\max }\right)$ in a semi-cell (i.e. half cell), under the assumption of a constant drift velocity. Even with small deviations from this assumption, as in the case of the DTs, the average of the meantimer distribution contains information about the average drift velocity in different regions of the cell, since it is computed using drift times produced by hits all over the gas volume. The mathematical expression of the meantimer relation depends on the track angle and on the pattern of cells hit by the track. In the trivial case the track crosses a semi-column of cells, i.e. the interested wires are at the same position for each couple of staggered cells, as shown in figure 6 . In this simple case the corresponding meantimer relation is

$$
T_{\max }=\left(t_{i}+t_{i+2}\right) / 2+t_{i+1}
$$

with $i=1,2$ for the two triplets of consecutive layers inside a superlayer. All the meantimer relations for different track angles and patterns of hit cells can be found in [8]. Also triplets of not adjacent layers inside a superlayer can be considered, this can be useful in case of unefficiencies in a given layer.

The proper meantimer formula is chosen track by track, using the direction and position information provided by the three-dimensional segments in a superlayer. This implies an iterative calibration procedure, starting with values of the drift velocity and of $t_{\text {trig }}$ that already result in efficient pattern recognition and segment reconstruction.

The meantimer is normally computed superlayer by superlayer, assuming the same effective drift velocity in all layers. It may be interesting, however, to calibrate the average drift velocity with finer granularity to take into account possible local variations within the layer quadruplet due to magnetic field inhomogeneities and to the variation of the track angle.

The mechanical precision of the wire and layer positions inside the superlayers is of the order of $100 \mu \mathrm{m}$ which corresponds to a bias of $1.8 \mathrm{~ns}$ on the measured drift times. This causes a different uncertainty on the $T_{\max }$ depending on the meantimer formula, the consequent error on the drift velocity is of the order of $1 \%$ or less. The uncertainty of the layer positions inside the superlayers should improve up to $10 \mu \mathrm{m}$ after the first alignment procedure which corresponds to an error on the drift velocity of $0.1 \%$ or less.

In the next paragraph the various steps of the drift velocity calibration procedure are listed. 
Calibration procedure. The calibration procedure of the drift velocity consists of the following steps.

- A Gaussian is fit to the meantimer distribution for each track pattern $j$ to estimate the mean value $T_{\max }^{j}$, the standard deviation $\sigma_{T}^{j}$, and the error on the mean $\sigma_{T}^{j} / \sqrt{N_{j}}$ (where $N_{j}$ is the number of entries in the distribution).

- The weighted average of the values of $T_{\max }^{j}$ is computed where the weights are taken as $N_{j} /\left(\sigma_{T}^{j}\right)^{2}$ :

$$
\left\langle T_{\max }\right\rangle=\frac{\sum_{j} \frac{T_{\max }^{j}}{\left(\sigma_{T}^{j}\right)^{2}} N_{j}}{\sum_{j} \frac{N_{j}}{\left(\sigma_{T}^{j}\right)^{2}}} .
$$

This accounts for the relative importance of the different cell patterns in the computation of the maximum drift time.

- Once $\left\langle T_{\max }\right\rangle$ is computed, it is straightforward to find the average drift velocity using the relation:

$$
v_{\text {drift }}=\frac{L / 2}{\left\langle T_{\max }\right\rangle}
$$

where $L$ is the width of the cell. The effective drift velocity computed for each superlayer is then stored in a database to be used by both the on-line and off-line hit reconstruction.

\subsection{Estimate of the cell resolution}

The meantimer technique allows the measurement of the cell resolution and hence the uncertainties on the reconstructed distance.

The standard deviation of the meantimer distribution $\left(\sigma_{T}^{j}\right)$ is a measurement of the resolution of $T_{\max }^{j}$. It can be therefore used to estimate the uncertainty on the measurement of the drift times $\left(\sigma_{t}^{j}\right)$ with a relation that depends on the particular formula used to compute the meantimer. For instance, in the case of tracks crossing a semi-column of cells, the time resolution can be computed as

$$
\sigma_{t}^{j}=\sqrt{\frac{2}{3}} \cdot \sigma_{T}^{j}
$$

which is valid under the assumption that the uncertainties are the same for all three layers used in the meantimer computation.

Since the cell resolution depends on the track angle, an average effective value is computed by averaging the different values obtained for the contributing cell patterns weighted on the number of entries in each meantimer histogram $\left(N_{j}\right)$ :

$$
\left\langle\sigma_{t}\right\rangle=\frac{\sum_{j} \sigma_{t}^{j} \cdot N_{j}}{\sum_{j} N_{j}} .
$$

The resolution of the reconstructed distance is therefore given by:

$$
\sigma_{d}=v_{\text {drift }} \cdot\left\langle\sigma_{t}\right\rangle
$$


This value is used during the reconstruction to assign the uncertainties to the hit positions in the gas volume. These uncertainties include the effect of the cell non-linearities (as those shown in figure 4) only on average, therefore their dependence on the distance from the wire cannot be taken into account with this method.

\section{Interplay of meantimer computation and time pedestals determination}

Reconstruction using a constant drift velocity requires both the calibration of the time pedestals needed for synchronization and of the average drift velocity. These two tasks are not independent since on one hand the computation of the meantimer requires knowledge of the time pedestals and on the other hand fine tuning of $t_{\text {trig }}$ is based on analysis of the residuals, which are directly affected by a mis-calibration of the drift velocity.

If the determination of $t_{\text {trig }}$ is affected by a systematic shift $\Delta t$ :

$$
t_{\text {trig }}^{\prime}=t_{\text {trig }}+\Delta t
$$

the meantimer will be consequently biased by a quantity that depends on the particular formula. In the case of tracks crossing a semi-column we can evaluate the effect on $T_{\max }$ as

$$
T_{\max }^{\prime}=T_{\max }-2 \Delta t
$$

In a simplified scenario, where this particular pattern is the one determining the meantimer calculation $\left(\left\langle T_{\max }\right\rangle \approx T_{\max }^{\prime}\right.$ ), the bias on $t_{\text {trig }}$ determination will result in a mis-calibration of the drift velocity $\Delta v_{\text {drift }}$, which can be estimated as

$$
\begin{aligned}
v_{\text {drift }}+\Delta v_{\text {drift }} & =\frac{L}{2 \cdot T_{\max }^{\prime}} \\
& =\frac{L}{2 \cdot\left(T_{\max }-2 \Delta t\right)} .
\end{aligned}
$$

To first order, this is equivalent to the following requirement:

$$
2 v_{\text {drift }} \Delta t-T_{\max } \Delta v_{\text {drift }}=0
$$

which can be considered as a calibration condition: all values of drift velocity and time pedestal that satisfy this relation will not affect the mean value of the residuals. This is strictly true only for small variations around the "optimal" values of $t_{\text {trig }}$ and $v_{\text {drift }}$ since larger fluctuations may affect pattern recognition efficiency and segment building. Lacking an external system for the track measurement, the segment is used as a reference for the computation of the residuals of the reconstructed drift distance.

The main sources of uncertainty in the determination of the time pedestal are the fluctuations in the mean value $\langle t\rangle$ and in the $\sigma$ of the fit in the different layers of a superlayer. The intrinsic statistical error, the presence of noise before the drift time box (evidenced, e.g., by the entries shown in figure 3 before the starting point of the drift time box), the finite step size of the TDC $(0.78 \mathrm{~ns})$ and the fact that the distribution is not perfectly described by eq. (2.1) limit the accuracy of $t_{\text {trig }}$ determination to about $1 \mathrm{~ns}$. Further systematic uncertainties come from the uncertainty 
of the drift velocity, as demonstrated by eq. (4.4), therefore higher accuracy can only be achieved using a procedure for fine tuning of the time pedestal which is independent of the drift velocity.

An alternative approach consists in using the different dependences on $t_{\text {trig }}$ mis-calibration of the various meantimer formulas to calibrate the pedestal. The differences among the values of $T_{\max }$ computed using different formulas can be used to measure the value of the mis-calibration $\Delta t$ once the dependence of the meantimer on the track impact angle is well understood. This would allow $t_{\text {trig }}$ to be tuned without relying on the residual distribution and therefore without depending on the calibration precision of the drift velocity. This alternative approach will be investigated in the future.

\section{Conclusions}

The calibration task is fundamental to the DT hit reconstruction: the knowledge of the time pedestal is an unavoidable prerequisite for the computation of the drift distance, while the calibration of the average drift velocity determines the accuracy of the reconstruction.

For this reason, a robust calibration procedure has been developed to satisfy the requirements imposed by all possible running conditions: dedicated cosmic runs, test beams, and ppcollision data.

The calibration algorithms described in the present document have been tested both on simulated and real data acquired during the 2004 test beam, the 2006 Magnet Test and Cosmic Challenge $[9,10]$ and the commissioning with cosmics.

Using the tools developed for the calibration and synchronization procedure we also studied the effect of possible mis-calibration of the pedestals and of the drift velocity on the muon track fit and thus eventually on higher level reconstructed quantities. We analyze these systematic uncertainties in the study of the physics reach of the experiment [11].

Further optimization is still possible. In particular, the accuracy of the current procedure is limited by the interdependence of the time pedestal and the drift velocity used in the reconstruction. Other methods for fine tuning of $t_{\text {trig }}$ are under study; a procedure based on the usage of different meantimer formulas to estimate the best value of the time pedestal is the most promising.

\section{References}

[1] CMS collaboration, CMS, the muon project: technical design report, CERN-LHCC-97-032.

[2] CMS collaboration, CMS, the magnet project: technical design report, CERN-LHCC-97-010.

[3] J. Puerta-Pelayo et al., Parameterization of the response of the muon barrel drift tubes, CMS-NOTE-2005-018.

[4] R. Veenhof, Garfield, a drift-chamber simulation program user's guide, CERN Program Library W5050.

[5] F. Cavallo et al., Test of MB3 muon barrel drift chamber with cosmic rays, CMS-NOTE-2003-017.

[6] C. Autermann et al., Test beam analysis of the first CMS MB2 drift tube muon chamber, CMS-NOTE-2003-007.

[7] RD5 collaboration, M. Andlinger et al., Bunch crossing identification at LHC using a mean timer technique, Nucl. Instrum. Meth. A 336 (1993) 91. 
[8] N. Amapane et al., Offline calibration procedure of the drift tube detectors, CMS-NOTE-2007-034.

[9] CMS collaboration, D. Lazic, The CMS Magnet Test and Cosmic Challenge (MTCC): operational experience and lessons learnt, Nucl. Phys. Proc. Suppl. 172 (2007) 3.

[10] S. Bolognesi et al., Measurement of drift velocity in the CMS barrel muon chambers at the CMS magnet test cosmic challenge, CMS-NOTE-2008-003.

[11] CMS collaboration, CMS physics: technical design report, volume II: physics performance, CERN-LHCC-2006-021. 